\title{
Quiloperitoneo espontáneo en el embarazo
}

\author{
Spontaneous chyloperitoneum in pregnancy \\ Juan Manuel Burgos¹, Diana Marcela Páez², María Alejandra Hincapié3, \\ Paula Andrea Fernández ${ }^{4}$, María Fernanda Escobar ${ }^{1}$ \\ ' Departamento de Ginecología y Obstetricia, Unidad de Alta Complejidad Obstétrica, Fundación Valle del Lili, Santiago de Cali, \\ Colombia \\ 2 Departamento de Ciencias de la Salud, Facultad de Medicina, Universidad ICESI, Santiago de Cali, Colombia \\ Médica Residente, Departamento de Ginecología y Obstetricia, Universidad ICESI, Santiago de Cali, Colombia \\ 4 Centro de Investigaciones Clínicas, Departamento de Ginecología y Obstetricia, Fundación Valle del Lili, Cali, Colombia
}

\section{Resumen}

Introducción. El quiloperitoneo consiste en la presencia de fluido linfático en la cavidad intraabdominal, complicación poco descrita en el embarazo y cuyas repercusiones no están bien establecidas.

Reporte de caso. Se presenta el caso de una mujer gestante con embarazo de 36,5 semanas, sin comorbilidades, hospitalizada por presentar cifras tensionales elevadas asociadas a síntomas de encefalopatía hipertensiva y bradicardia fetal sostenida; se le practicó una cesárea urgente en la cual se evidenció corioperitoneo incidental. La tomografía computarizada abdominal de control no demostró alteraciones.

Discusión. Se descartaron las principales causas del quiloperitoneo, las cuales son neoplasia maligna y trauma. Se consideró que se trataba de un quiloperitoneo espontáneo posiblemente relacionado con la congestión pélvica propia del embarazo, por vasodilatación secundaria al influjo hormonal, aumento de la presión intraabdominal y contribución del volumen ascítico por la preeclampsia, que genera ruptura traumática de los vasos linfáticos.

Conclusión. La ascitis quilosa es una entidad poco frecuente que puede verse asociada con el embarazo. Es fundamental el criterio acertado del ginecoobstetra para determinar tempranamente la etiología.

Palabras clave: embarazo; hipertensión inducida en el embarazo; peritoneo; ascitis quilosa; factores de riesgo; cesárea.

Fecha de recibido: 6/03/2019 - Fecha aceptación: 2/05/2019

Correspondencia: Juan Manuel Burgos, Fundación Valle del Lili, Carrera 98 N 18-49, Cali, Colombia. Teléfono: (572) 331-9090, extensión: 3049 a 3055; fax: (572) 331-9090

Correo electrónico: juanmaburgos28@hotmail.com

Citar como: Burgos JM, Diana PM, Hincapié MA, Fernández PA, Escobar MF. Quiloperitoneo espontáneo en embarazo. Rev Colomb Cir. 2020;35:119-22. https://doi.org/10.30944/20117582.597

Este es un artículo de acceso abierto bajo una Licencia Creative Commons - BY-NC-ND https://creativecommons.org/licenses/by-ncnd/4.0/deed.es 


\begin{abstract}
Introduction: Chyloperitoneum consists of the presence of a lymphatic fluid in the intra-abdominal cavity, a complication little described during pregnancy, and whose repercussions are not well established.
\end{abstract}

Case report: A pregnant woman with a pregnancy of 36.5 weeks, without comorbidities, is hospitalized in the context of high blood pressure levels associated with symptoms of hypertensive encephalopathy and sustained fetal bradycardia, leading to an emergent cesarean section where incidental chorioperitoneum was evidenced. Posterior abdominal CT control showed no alterations.

Discussion: The main associated etiologies (malignancy and trauma) were ruled out. It is considered a presentation of spontaneous chyloperitoneum that could be related to the pelvic congestion of pregnancy due to: vasodilatation secondary to hormonal influence, increased intra-abdominal pressure and contribution of ascites due to preeclampsia, generating traumatic rupture of lymphatic vessels.

Conclusion: Chylous ascites is a rare entity that can be associated with pregnancy. It is essential that gynecologist and obstetrician correctly approach these patients to determine the etiology early..

Keywords: pregnancy; hypertension, pregnancy-induced; peritoneum; chylous ascites; risk factors; cesarean section.

\section{Introducción}

La ascitis quilosa, o quiloperitoneo, consiste en la presencia de fluido linfático en la cavidad intraabdominal I. Se produce cuando existe una lesión del sistema linfático cuyas causas pueden ser diversas ${ }^{2 .}$ En casi el 6o \% de los casos, es secundario a cáncer abdominal o cirrosis y, en los países de bajos y medianos ingresos, a infecciones por micobacterias o parásitos ${ }^{3}$. Es una complicación que rara vez ocurre en el embarazo, poco descrita y cuyas repercusiones clínicas no están bien establecidas.

Se presenta el caso de una mujer con embarazo de 36,5 semanas, con evidencia de quiloperitoneo como hallazgo incidental durante la cesárea de urgencia.

\section{Caso clínico}

Se trata de una paciente de 23 años de edad con embarazo de 36,5 semanas de gestación, con antecedentes de dos embarazos y una cesárea, y sin comorbilidades conocidas. Consultó a otra institución por un cuadro clínico de Io horas de evolución consistente en ausencia de movimientos fetales, cefalea global y epigastralgia intensa; presentaba hipertensión arterial sistémica (I40/90 $\mathrm{mm} \mathrm{Hg}$ ) y no auscultaban fetocardia.
Consideraron el diagnóstico de preeclampsia grave con alta sospecha de óbito fetal, por lo cual le administraron sulfato de magnesio y una dosis única de nifedipino, y la remitieron a nuestra institución.

$\mathrm{Al}$ ingreso, la paciente presentaba síntomas de encefalopatía hipertensiva con cifras tensionales elevadas ( $145 / 95 \mathrm{~mm} \mathrm{Hg}$ ) y bradicardia fetal sostenida (frecuencia cardiaca fetal: IIo latidos por minuto).

La puntuación en el índice de BISHOP fue desfavorable y se indicó una cesárea de urgencia, bajo estrategias de reanimación fetal in utero y manejo de la preeclampsia grave según el protocolo institucional, con sulfato de magnesio y vasodilatador oral y endovenoso.

$\mathrm{Al}$ ingresar a la cavidad abdominal durante la cirugía, se obtuvieron $200 \mathrm{ml}$ de líquido blanquecino, inodoro y de aspecto turbio, del cual se tomó una muestra para cultivo. Se obtuvo un recién nacido sano de sexo masculino, con peso de 2,805 g y puntaje de 9-9-Io en la prueba de Apgar, al minuto, 5 minutos y Io minutos de haber nacido.

Debido al hallazgo de líquido peritoneal lechoso, se le solicitó al Servicio de Cirugía General una revisión exhaustiva de la cavidad abdominal; 
no se evidenciaron lesiones aparentes y llamó la atención la dilatación de los vasos linfáticos en el tercio proximal del íleon. Además, el apéndice cecal estaba edematoso, eritematoso y con ubicación proximal subserosa, por lo cual se practicó una apendicectomía con lavado peritoneal, sin que se presentaran complicaciones. Se enviaron muestras para análisis microbiológico y citoquímico, y se administró ceftriaxona más metronidazol por una presunta infección asociada. En el estudio del líquido peritoneal se reportó: aumento de los triglicéridos, 42I mg/dl; glucosa, $88,8 \mathrm{mg} / \mathrm{dl}$; deshidrogenasa láctica, I.I95 UI/L; proteínas, 3,8 g/dl; albúmina, I,74 g/dl; recuento celular, con segmentados, y coloración de Gram sin bacterias ni polimorfonucleados.

Estos hallazgos fueron muy sugestivos de quiloperitoneo. La tomografía computarizada (TC) abdominal con contraste, solicitada para descartar un proceso neoplásico abdominal o retroperitoneal, no demostró alteraciones que pudieran explicar el quiloperitoneo.

El estudio de histopatología no demostró apendicitis y, en el cultivo del líquido peritoneal para bacterias aerobias, se obtuvo Staphylococcus lugdunensis, perteneciente a la flora normal de la piel.

La evolución posquirúrgica fue normal, las cifras tensionales permanecieron dentro del rango normal mediante esquema terapéutico por vía oral, y no hubo signos clínicos ni elevación de reactantes de fase aguda que sugirieran una infección activa.

Ante la evolución satisfactoria se consideró que se trataba de un quiloperitoneo espontáneo de origen idiopático y se decidió autorizar el egreso con antihipertensivos orales y advertencia de regresar a consulta si se presentaban signos de alarma. En la consulta de control un mes después, la paciente se encontraba en buenas condiciones, con los cambios normales del puerperio.

\section{Discusión}

La ascitis quilosa es la acumulación de quilo en la cavidad peritoneal. La presencia de quilo en el líquido peritoneal se confirma por la elevación de la concentración de triglicéridos, por lo general, mayor de $200 \mathrm{mg} / \mathrm{dl}^{4}$. Se han identificado tres mecanismos fisiopatológicos asociados. El primero corresponde a la obstrucción del flujo linfático secundaria a procesos malignos, con mayor frecuencia (50\%), un linfoma ${ }^{5}$. El segundo mecanismo es la exudación de linfa a través de los vasos retroperitoneales sin válvulas funcionales. El tercero es la obstrucción del conducto torácico, secundaria a un trauma, que ocasiona la salida de quilo hacia la cavidad abdominal 5 .

Se han descrito casos de quiloperitoneo de origen traumático, como una complicación rara de las cirugías ginecológicas, que se relaciona usualmente con la disección extensa de los ganglios linfáticos ${ }^{6,7}$, y cuya incidencia aproximada es menor del $2 \%{ }^{8}$.

Se han identificado otras posibles causas menos frecuentes de ascitis quilosa, como cirrosis (I \% $)^{5}$, infecciones de gran prevalencia en países de bajos y medianos ingresos, tuberculosis peritoneal, filariasis, malformaciones congénitas de los vasos linfáticos, vasculitis y lesiones autoinmunitarias. Sin embargo, la ascitis quilosa de origen congénito, generalmente, se inicia de forma aguda en la infancia ${ }^{8}$. Su pronóstico depende de la causa que la origine 9.

En el caso expuesto, no existía ninguna de estas posibles causas, las cuales se descartaron mediante TC abdominal con contraste y exámenes de laboratorio, incluidos los cultivos para micobacterias. En pocos reportes anteriores se ha mencionado que la congestión pélvica propia del embarazo, debida a la vasodilatación secundaria al influjo hormonal de progesterona y al aumento de la presión intrabdominal por el útero grávido, puede causar ruptura traumática de los vasos linfáticos ${ }^{3}$.

Thompson, et al., reportaron una paciente primigestante con un embarazo de 35 semanas de gestación y preeclampsia grave, a quien se le practicó la cesárea por sospecha de desprendimiento placentario, cirugía en la cual se encontró un quiloperitoneo de $800 \mathrm{ml}$. Los autores postularon como su origen, la ruptura de vasos linfá- 
ticos pélvicos por compresión del útero grávido, agravada esta última por el volumen del líquido ascítico libre producido por la preeclampsia ${ }^{4}$. Hay autores que vinculan el desarrollo de ascitis con la preeclampsia durante el embarazo; se ha relacionado con la presencia de hipoproteinemia y disminución del gradiente entre la albúmina y la globulina ${ }^{\mathrm{IO}}$.

El presente reporte tiene grandes similitudes con el de Thompson ${ }^{4}$. Es interesante mencionar que, aunque se proponga una ruptura espontánea de los vasos linfáticos, generalmente, no se observa el lugar de la fuga de quilo; además, la ascitis quilosa parece autolimitarse después de la descompresión de la cavidad abdominal. Este comportamiento favorece la hipótesis de que la forma idiopática se podría presentar por un aumento anormal de la congestión pélvica, asociado con una alteración de los conductos linfáticos en algunos pacientes. En el presente caso, no fue posible complementar el estudio para confirmar o descartar posibles variantes anatómicas del sistema linfático.

Según la revisión bibliográfica realizada para este informe, este caso es el séptimo reportado y el tercero no asociado con pancreatitis o masas intraabdominales, el cual se evidenció como un hallazgo incidental durante la cesárea.

\section{Conclusión}

La ascitis quilosa es una entidad poco frecuente que puede verse asociada con el embarazo. Es fundamental que el ginecoobstetra conozca el enfoque diagnóstico de estos casos para determinar, de ser posible de forma temprana, la causa etiológica de enfermedades complejas y, muchas veces, curables. La ascitis quilosa, sin causa aparente, parece ser una entidad benigna que se resuelve espontáneamente durante el puerperio.

\section{Consideraciones éticas}

Consentimiento informado: Este reporte se basa en una revisión retrospectiva de la histo- ria clínica, por lo cual no hay necesidad de un consentimiento informado. El Comité de Ética Institucional aprobó el diseño y la metodología del estudio.

Conflicto de intereses: ninguno de los autores presenta conflicto de intereses.

Fuentes de financiación: recursos propios de los autores.

\section{Referencias}

I. Miller H, Anderson M, Smith C, Shamshirsaz A, Fox K. Chylous ascites complicating modified radical hysterectomy for placenta percreta. Obstet Gynecol. 2016;128:973-5. https://doi.org/IO.IO97/ aog.00ooooooooool562.

2. Sun L, Wu H, Zhuang Y, Guan Y. A rare case of pregnancy complicated by mesenteric mass: What does chylous ascites tell us? World J Gastroenterol. 2007;13:1632-5. https://doi.org/I0.3748/wjg.vi3.iro.I632.

3. Babic I, Tulbah M, Ghourab S. Spontaneous resolution of chylous ascites following delivery: A case report. J Med Case Rep. 20I2;6:I87. https://doi.org/IO.II86/I752I947-6-I87.

4. Thompson K, Khabbaz A. Incidental chylous ascites at the time of cesarean section. Case Rep Obstet Gynecol. 2015;2015:530210. https://doi.org/IO.II55/2015/5302IO.

5. Almakdisi T, Massoud S, Makdisi G. Lymphomas and chylous ascites: Review of the literature. Oncologist. 2005;I0:632-5. https://doi.org/I0.I634/theoncologist.Io-8-632.

6. Tulunay G, Ureyen I, Turan T, Karalok A, Kavak D, Ozgul N. Chylous ascites: Analysis of 24 patients. Gynecol Oncol. 2012;I27:I9I-7. https://doi.org/IO.IOI6/j. ygyno.2012.06.023.

7. Zhao Y, Hu W, Hou X, Zhou Q. Chylous ascites after laparoscopic lymph node dissection in gynecologic malignancies. J Minim Invasive Gynecol. 20I4;2I:90-6. https://doi.org/I0.I0I6/j.jmig.2013.07.005.

8. Jiménez C. Ascitis quilosa. Presentación de un caso y revisión de la fisiopatología. Rev Colomb Cir. 2004;19:76-84.

9. Pamo O, Caballero J, Alfaro J. Quiloperitoneo como forma de presentación del linfoma intestinal difuso: reporte de un caso. Rev Medica Hered. 2007;17:155-60. https://doi.org/20453/rmh.vi8i3.924.

Io. Chawla S, Kumar B, Bhalla M. Case of ascites in preeclampsia. Med J Armed Forces India. 2012;68:257-9. https://doi.org/IO.IOI6/j.mjafi.20I2.04.003. 